\title{
Design and validation of an angle-resolved low-coherence interferometry fiber probe for in vivo clinical measurements of depth-resolved nuclear morphology
}

\author{
Yizheng Zhu, ${ }^{a}$ Neil G. Terry, ${ }^{a}$ John T. Woosley, ${ }^{\mathrm{b}}$ Nicholas J. Shaheen, ${ }^{\mathrm{c}}$ and Adam Wax ${ }^{\mathrm{d}}$ \\ a Duke University, Department of Biomedical Engineering, 136 Hudson Hall, Durham, North Carolina 27708 \\ bUniversity of North Carolina, Department of Pathology and Laboratory Medicine, Campus Box 7525, Chapel Hill, \\ North Carolina 27599 \\ 'University of North Carolina, School of Medicine, Center for Esophageal Diseases and Swallowing, 4102 \\ Bioinformatics, Campus Box 7080, Chapel Hill, North Carolina 27599 \\ ${ }^{\mathrm{d}}$ Duke University, Department of Biomedical Engineering, 136 Hudson Hall, Durham, North Carolina 27708
}

\begin{abstract}
We present a novel Fourier-domain angle-resolved low-coherence interferometry $(\mathrm{a} / \mathrm{LCl})$ fiber probe designed for in vivo clinical application in gastrointestinal endoscopy. The a/LCl technique measures the depthresolved angular scattering distribution to determine the size distribution and optical density of cell nuclei for assessing the health of epithelial tissues. Clinical application is enabled by an endoscopic fiber-optic probe that employs a 2.3-m-long coherent fiber bundle and is compatible with the standard 2.8-mm-diam biopsy channel of a gastroscope. The probe allows for real-time data acquisition by collecting the scattering from multiple angles in parallel, enabled by the Fourier domain approach. The performance of the probe is characterized through measurement of critical parameters. The depth-resolved sizing capability of the system is demonstrated using single- and double-layer microsphere phantoms with subwavelength sizing precision and accuracy achieved. Initial results from a clinical feasibility test are also presented to show in vivo application in the human esophagus. (C) 2011 Society of Photo-Optical Instrumentation Engineers (SPIE). [DOI: 10.1117/1.3520130]
\end{abstract}

Keywords: low coherence interferometry; angle resolved; endoscopic imaging; clinical application; Barrett's esophagus.

Paper 09465SSR received Oct. 13, 2009; revised manuscript received Jan. 14, 2010; accepted for publication Feb. 4, 2010; published online Jan. 10, 2011.

\section{Introduction}

Barrett's esophagus (BE) is a metaplastic transformation of the epithelial lining of the lower esophagus and is considered a premalignant condition associated with a markedly increased risk of esophageal adenocarcinoma. ${ }^{1}$ Early detection of dysplasia in $\mathrm{BE}$ patients requires long-term periodic surveillance by means of standard white-light endoscopy with stepwise four quadrant biopsies followed by histological analysis. ${ }^{2}$ This approach is the current standard of care but is invasive and provides only limited examination of tissues, with high likelihood of sampling error due to the random nature of endoscopic biopsies.

Over the past decade, new optical techniques, such as fluorescence endoscopy ${ }^{3}$ and narrowband imaging, ${ }^{4,5}$ have been developed as adjuncts to the standard approach to enhance the contrast of wide field imaging and improve visual identification of dysplastic tissues. These approaches could help reduce the number of biopsies yet are still invasive because tissue removal is required.

In vivo optical biopsy, in contrast, allows for noninvasive assessment of tissue health. A number of advanced techniques have demonstrated potential as promising clinical tools in assisting physicians with timely and accurate diagnosis. ${ }^{6-8}$ Light-scattering spectroscopy (LSS), ${ }^{9}$ for instance, obtains

Address all correspondence to: Adam Wax, Duke University, Department of Biomedical Engineering, 136 Hudson Hall, Durham, North Carolina 27708. Tel: 919-660-5143; Fax 919-684-4488; E-mail: a.wax@duke.edu. quantitative information of nuclear morphology by extracting the singly backscattered light from epithelial layers of tissue using modeling. LSS has been shown effective for in vivo identification of precancerous cells in a variety of epithelial tissues using the population density and percentage of enlarged cell nuclei as biomarkers $;^{10-12}$ although, certain aspects of the approach have recently been called into question. ${ }^{13}$ Elastic scattering spectroscopy [(ESS), also known as diffuse reflectance spectroscopy], on the other hand, analyzes mainly multiply scattered light, which is sensitive to bulk tissue scattering properties as well as absorption by hemoglobin. ESS has been applied to the detection of dysplastic lesions in the gastrointestinal (GI) tract. ${ }^{14,15}$ Techniques based on Raman spectroscopy also show promise for diagnosis of dysplasia in BE patients. ${ }^{16,17}$ To enable in vivo application of these spectroscopic techniques, numerous fiber-optic probes have been proposed and developed. ${ }^{18}$

In addition to spectroscopic approaches, in vivo optical imaging systems, such as confocal fluorescence endomicroscopy (CFM) and optical coherence tomography (OCT), have received considerable attention for GI applications, aiming to provide real-time histology of tissues. CFM probes, based on either a single scanning fiber ${ }^{19-21}$ or a fiber bundle, ${ }^{22-24}$ have both been implemented for high-resolution imaging in the GI tract and are commercially available today. As an

1083-3668/2011/16(1)/011003/10/\$25.00 @ 2011 SPIE 


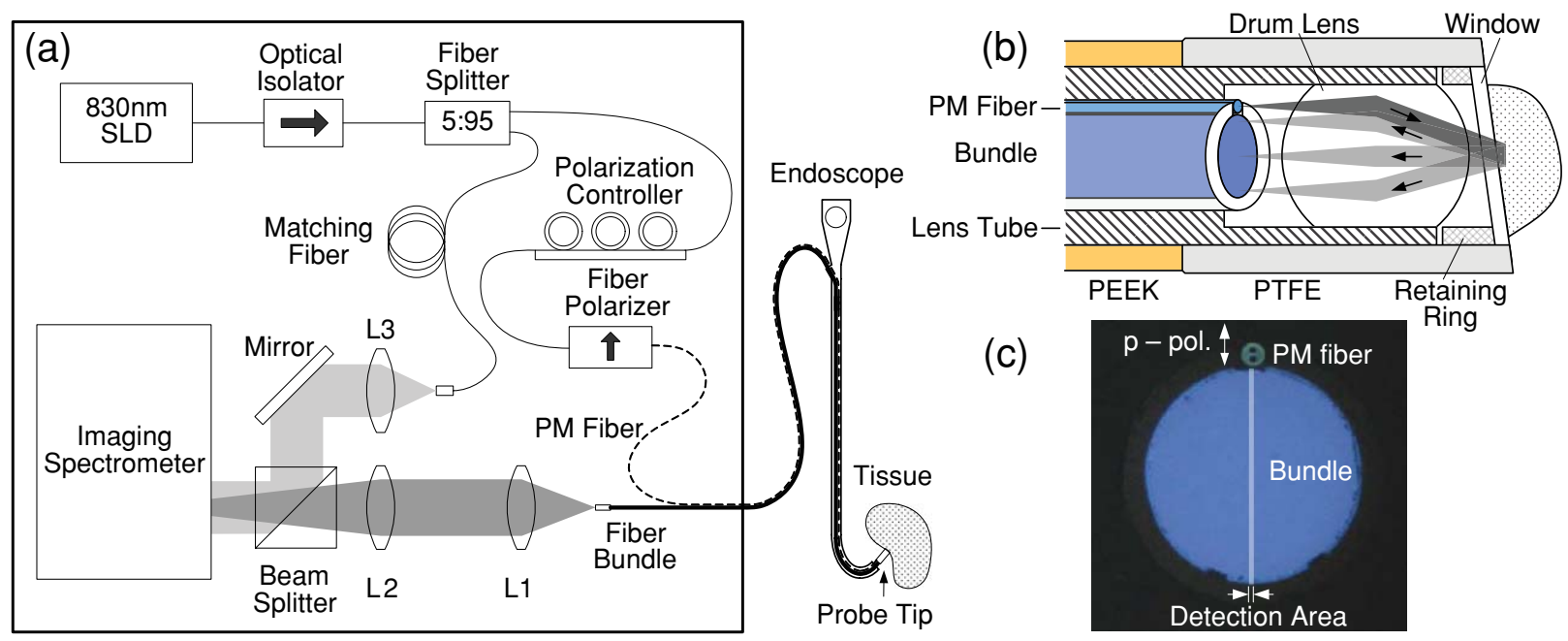

Fig. 1 Clinical Fourier-domain a/LCl system: (a) System configuration, (b) probe tip assembly showing sample illumination and scattering detection with fiber bundle (in-plane tilt of optical window is for convenience of illustration), and (c) photograph of the fiber bundle and delivery PM fiber with detection area highlighted.

another approach with greater depth range but less lateral resolution, a number of OCT systems are able to inspect large tissue volumes in vivo and demonstrate diagnostic potential as well. ${ }^{25-28}$

Angle-resolved low-coherence interferometry (a/LCI) offers another means of optical biopsy, by combining the depth resolution of OCT with angular light-scattering measurements to obtain depth-resolved morphological and optical properties of cell nuclei. ${ }^{29}$ In a/LCI, light scattered by a sample at different angles is mixed with a reference field to produce the depthresolved angular scattering distribution of the sample, which is then subject to inverse light-scattering analysis based on Mie theory ${ }^{30}$ or T-matrix. ${ }^{31}$ With the incorporation of a coherent fiber bundle for parallel collection of light across a range of scattering angles, ${ }^{32}$ this approach was used for ex vivo detection of nuclear atypia in dysplastic BE. ${ }^{29,33}$

In this paper, we present the detailed design and validation of a Fourier-domain a/LCI system with a miniature endoscopic probe as a clinical device for in vivo evaluation of nuclear morphology in BE tissues. The design and fabrication of the probe, built with a $2.3-\mathrm{m}$ fiber bundle to fit into the $2.8-\mathrm{mm}$ accessory channel of a gastroscope with a standard working length of $1.05 \mathrm{~m}$, are described. Its optical performance is characterized in detail. Results from phantom testing and a pilot in vivo testing in BE patients are also presented.

\section{Instrumentation and Probe Design}

The Fourier-domain a/LCI system is based on a modified MachZehnder interferometer, as shown in Fig. 1(a). Light from an 830-nm superluminescent diode (SLD, Superlum Diodes, Moscow, Russia $\Delta \lambda_{\mathrm{FWHM}}=19 \mathrm{~nm}$ ) is split by a 5:95 fiber splitter into reference and sample arms. In the sample arm, a polarization controller adjusts the polarization to maximize the power transmission through an inline fiber polarizer (Chiral Photonics, Inc., Pine Brook, NJ). The polarizer is necessary because the angular scattering distribution depends critically on incident polarization, which must be known to establish accu- rate fitting models. In this system, p-polarized light is delivered to the sample by a polarization-maintaining (PM) fiber. A leached, coherent fiber bundle (Schott; length: $2.3 \mathrm{~m}$; imaging area: $1.1 \mathrm{~mm}$ diam; pixel count: 18,000) collects the scattering signal and transfers it to the proximal end. The signal is then mixed with the collimated reference field and detected by an imaging spectrograph (SpectraPro 2150i, Acton Research Corporation, Acton, MA) with a thermoelectrically cooled CCD (PIXIS:100, Princeton Instruments; Trenton, NJ $100 \times 1340$ $20 \mu \mathrm{m}$ pixels). Pathlength is matched by moving collimator L3. A 45-deg-angled mirror bends the light path to minimize system footprint. It is worth noting that although the delivery fiber is PM, the fiber bundle does alter the polarization of the collected scattering in transmission. The total intensity, however, is not changed and still follows the Mie model at the proximal end of the fiber bundle, where we found that the output polarization state tends to be approximately randomized. To collect all the energy, we place the matching fiber in the reference arm such that its output polarization is also randomized to maximize the collection efficiency of the sample signal. This renders the signal detection polarization insensitive.

Figure 1(b) shows the tip assembly of the endoscopic probe. The PM fiber and the fiber bundle are positioned in the focal plane of a miniature drum lens (diameter: $1.5 \mathrm{~mm}$; length: $2.5 \mathrm{~mm}$ ). The sample is illuminated by the collimated beam, and the angular scattering is converted by the lens to a spatial distribution at the bundle endface. A lens tube holds these components together using medical-grade UV adhesive. To keep the internal optics from contamination, we cover the tip with a protective cap that consists of a short Teflon ${ }^{\circledR}$ (PTFE) tube, an optical window made from No. 1 coverglass, and a back-side retaining ring. To avoid specular reflection, the window is tilted $8 \mathrm{deg}$ out of the incident plane. The inner surface of the Teflon tube is treated with a sodium-based etchant prior to assembly to maximize the bonding strength. In addition, the retaining ring further secures the optical window and enhances the tip's structural rigidity to withstand greater mechanical stress. The outer surface of the Teflon tube is left 

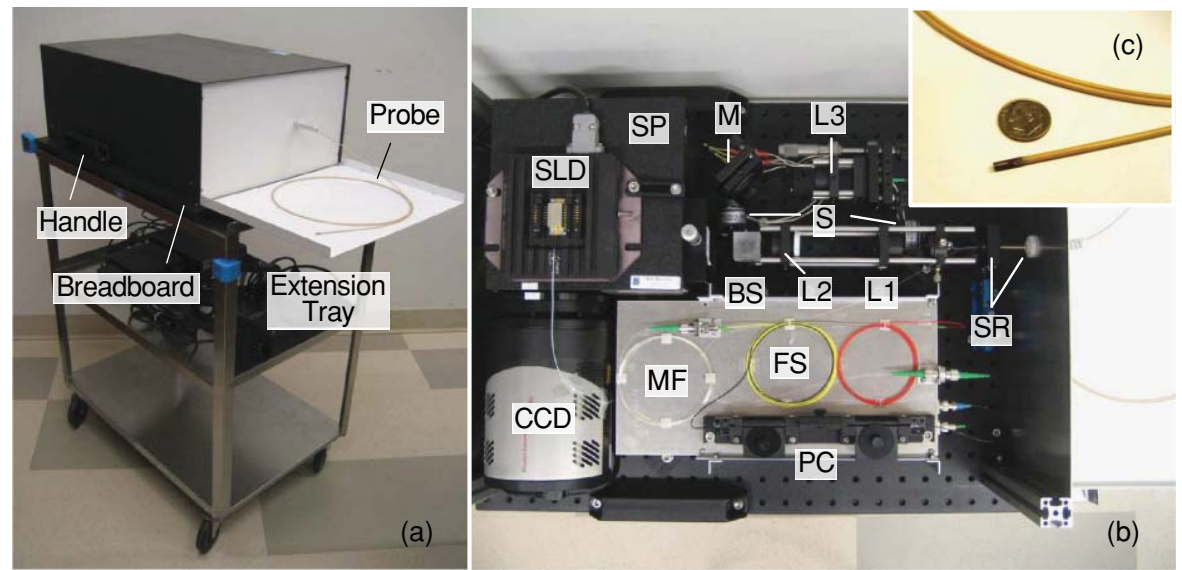

Fig. 2 Photographs of the clinical a/LCl system: (a) Enclosed system on the cart with the extension tray installed and (b) system components visible with top cover removed: SP-spectrometer; CCD-camera; SLD-light source; M-45-deg mirror; L3-reference arm collimator; S-shutter; BSbeamsplitter; L1\&L2-4f system ( $\mathrm{f} 1=40 \mathrm{~mm} ; \mathrm{f} 2=60 \mathrm{~mm}$ ); SR-bundle strain relief; MF-matching fiber; FS-fiber splitter; PC-polarization controller. (c) Close-up of the probe tip compared to a U.S. dime.

untreated to maintain its low friction coefficient for ease of passage through the accessory channel of the gastroscope. The rest of the probe is sheathed by a polyetheretherketone (PEEK) tubing (o.d.: $2.3 \mathrm{~mm}$ ), a material with balanced flexibility and mechanical strength.

The detectable scattering signal collected by the probe is limited to a narrow vertical strip by the entrance slit of the imaging spectrograph, as shown in Fig. 1(c). This image also shows that proper ambient illumination allows for visualization of the stress rods in the PM fiber, which is used for polarization alignment during fabrication.

The finished system, shown in Fig. 2, is built on a $24 \times 18$ in. aluminum breadboard and carried on a $27 \times 18$ in. stainless steel utility cart with four swivel wheels for maneuverability in the procedure room. During operation, the probe is placed on a detachable extension tray. The system footprint is minimized by a three-shelf rack, which houses most fiber-optic components.

\section{System and Probe Characterization}

Clinical measurement requires detailed characterization of the system. In this section, we evaluate the performance of this $\mathrm{a} / \mathrm{LCI}$ endoscopic probe and investigate the optical properties of the fiber bundle and their implications in interferometric measurement.

\subsection{Signal Processing}

The intensity detected at the $n$, th row of the CCD represents the spectrum of sample signal scattered at angle $\theta_{n}$, and can be written as

$$
\begin{aligned}
I\left(\lambda, \theta_{n}\right)= & I_{r}\left(\lambda, \theta_{n}\right)+I_{s}\left(\lambda, \theta_{n}\right) \\
& +2 \eta_{n} \sqrt{I_{r}\left(\lambda, \theta_{n}\right) I_{s}\left(\lambda, \theta_{n}\right)} \cos \left[\Delta \varphi_{\mathrm{sr}}\left(\lambda, \theta_{n}\right)\right],
\end{aligned}
$$

where $I_{\mathrm{s}}\left(\lambda, \theta_{n}\right)$ and $I_{\mathrm{r}}\left(\lambda, \theta_{n}\right)$ are the sample and reference field intensities at angle $\theta_{n}$, respectively; $\Delta \varphi_{\text {sr }}$ is the phase difference between them; $\eta_{n}$ is a system efficiency factor that is a combination of the interference efficiency and the coupling efficiency of the fiber probe, drum lens, and $4 \mathrm{f}$ imaging system; and $\lambda$ is the wavelength.

The signal processing begins with the subtraction of $I_{\mathrm{r}}\left(\lambda, \theta_{n}\right)$ and $I_{\mathrm{s}}\left(\lambda, \theta_{n}\right)$, which are recorded separately using two computercontrolled mechanical shutters in the reference and sample arms. Because $I_{\mathrm{r}}\left(\lambda, \theta_{n}\right)$ is relatively stable, it is saved only once at the beginning of each data acquisition session. $I_{\mathrm{s}}\left(\lambda, \theta_{n}\right)$, however, is measured immediately prior to every acquisition of the combined field to minimize field fluctuation in the subtraction. The highest acquisition rate of the system is $2.5 \mathrm{~Hz}$, limited by the shutter speed, which can be improved by the use of fiber-optic switches.

After subtraction, the interferometric term is resampled into wavenumber domain, Fourier transformed, and normalized to yield a depth-resolved angular intensity distribution, ${ }^{32}$ which is subsequently analyzed at each depth using Mie theory model for size determination.

\subsection{Angular Range}

We first measure the probe's angular range, an important parameter for accurate analysis of light-scattering data. Using a rotating mirror as shown in Fig. 3(a), the scattering angle $\theta$ is found to be linearly dependent on the CCD pixel position $N$. The collimated illumination beam is reflected by the mirror and focused onto the fiber bundle while the mirror rotates. For convenience, we define $\theta$ as the compliment of the conventionally defined scattering angle (i.e., $\theta=0 \mathrm{deg}$ for backscattering instead of $180 \mathrm{deg}$ ).

Figure 3(a) shows superposed images of multiple focused spots that are observed as the mirror rotates in steps of $0.035 \mathrm{rad}(2 \mathrm{deg})$ for a range of $0.56 \mathrm{rad}(32 \mathrm{deg})$. The position of the focused spot relative to the optical axis $h$, which has a linear dependence on $N$, is related to $\theta$ by

$$
\begin{aligned}
\tan \left(\theta_{\text {axis }}-\theta\right) & =\frac{h}{f} \Rightarrow \theta \approx \theta_{\text {axis }}-\frac{h}{f} \\
& =\theta_{0}+\alpha\left(N-N_{\text {low }}\right), N \in\left[N_{\text {low }}, N_{\text {hi }}\right],
\end{aligned}
$$



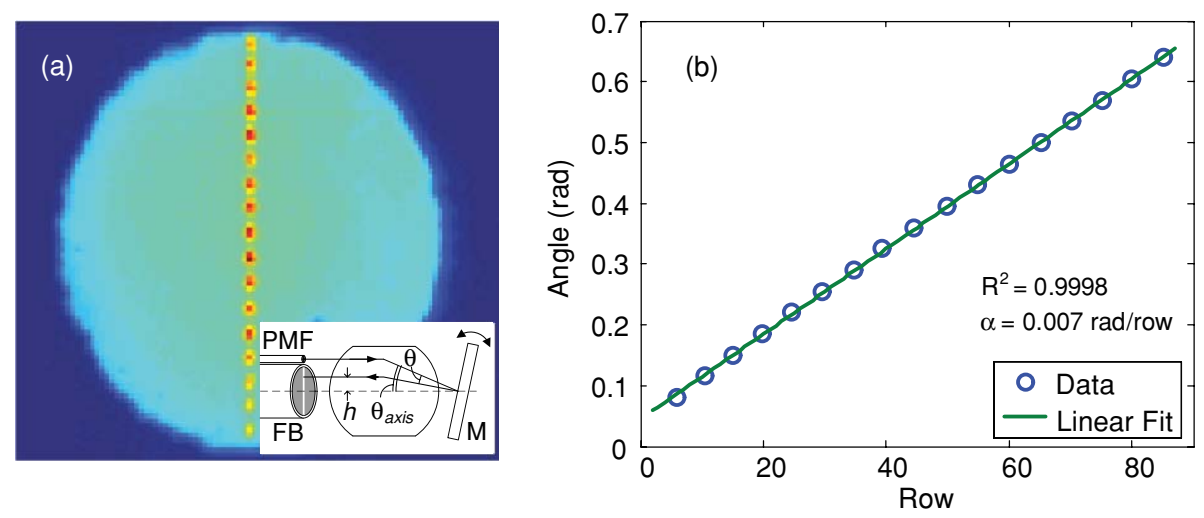

Fig. 3 Rotating mirror experiment: (a) Superposed images of fiber bundle and focused spots; (inset) schematics of the experiment: PMF-polarization maintaining fiber; FB-fiber bundle; M-mirror. (b) Position of the focused spots versus mirror angle.

where $\theta_{\text {axis }}$ corresponds to the angle of backscattering along the optical axis, $f$ is the focal length of the drum lens, $\alpha$ is the angular step per CCD row or angular resolution, $\theta_{0}$ is the lowest angle that can be received by the bundle, and $\left[N_{\text {low }} N_{\text {hi }}\right]$ is the pixel range of the bundle on the CCD. The $\pm 0.28 \mathrm{rad}$ (16 deg) range collected by the probe resides within the small angle approximation, which enables a good linear approximation of $\tan (\theta)=\theta$ and, hence, the linear dependence on $N$. Figure 3(b) plots the center position of the spots versus angle, confirming the linearity with an angular resolution of $0.007 \mathrm{rad} /$ row. $\theta_{0}$ can be calculated as $\delta / f$ by analyzing Fig. 1(c), where $\delta$ is the distance from the PM fiber core to the upper edge of the bundle. The full angular range of the bundle is found to be $0.08-0.67 \mathrm{rad}$. For signal processing, we use a reduced range of $0.1-0.55$ rad to remove edge effects and weak signals at the higher end of the range.

We note that, because the mirror experiment is carried out in air, these angles differ from those in other samples, such as tissue and phantoms, due to Snell's law by a factor of $1 / n$ with $n$ being the sample's refractive index. In our fitting models, the angles are always scaled to the equivalents in air.

\subsection{Depth Range}

The achieved probing depth is a critical parameter for the clinical a/LCI probe. It is not only dependent on signal falloff, but more importantly related to its angular range. We examine the impact of sample depth on angular range and scattering intensity by repeating the mirror experiment at various offsets (depths) between the mirror and the optical window. Figure 4(a) shows the change of spot position for four angles as the mirror is progressively moved from 150 to $750 \mu \mathrm{m}$ beyond the optical window. The spot position remains nearly the same with a standard deviation of 0.6 pixel $(0.004 \mathrm{rad})$, or $0.7 \%$ full scale. We therefore conclude that the angular range remains constant over this range of depth.

In Fig. 4(b), we also plot the signal intensity trend throughout the depth. At each angle, the intensity falls off as a function of depth due to beam divergence and lens aberration, which reduce back-coupling efficiency. Also, light intensity at higher angles experiences a faster decline as a consequence of the oblique incidence, as illustrated in Fig. 4(b). For scattering location 1, the high angle reflection is well received. At a deeper location 2 , however, the reflection at the same angle is shifted
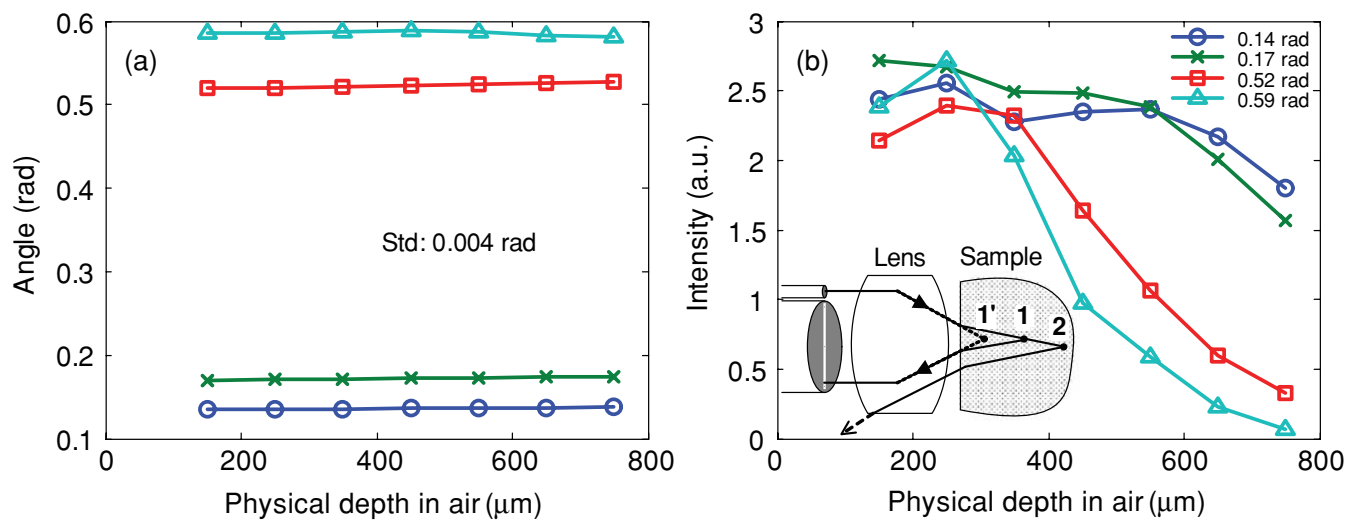

Fig. 4 The impact of sample depth on detected signal: (a) Angular range remains constant throughout the examined depth range for both high and low angles and (b) sample intensity declines with depth faster at high angles due to limited lens collection aperture. 


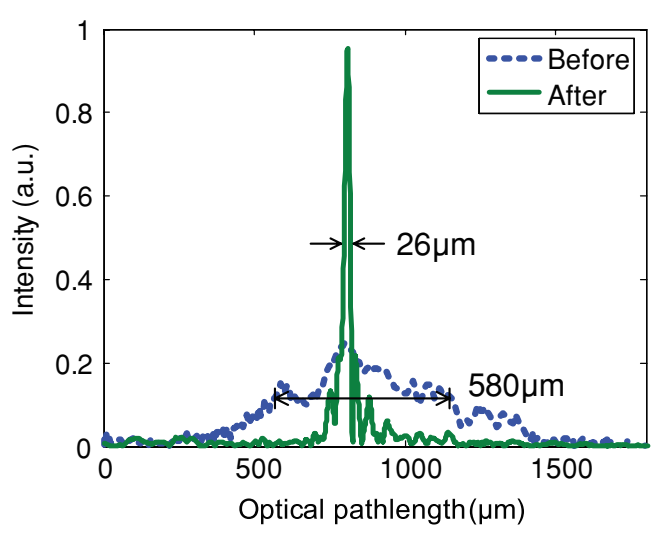

Fig. 5 FWHM of a mirror reflection before $(580 \mu \mathrm{m})$ and after $(26 \mu \mathrm{m})$ dispersion compensation.

downward due to the oblique incident and therefore becomes uncollectable by the lens. Scattering from these deep sites is still detectable but with a reduced angular range. The depth dependence of the maximum detectable angular range, $\theta_{\max }$, can be mathematically written as

$\theta_{\max }=\left\{\begin{array}{cl}\frac{2 r+\delta}{f}(\text { full range }), & d \leq n\left(\frac{R+r+\delta}{2 r+\delta} f+d_{0}\right) \\ \max \left(\frac{R+r+\delta}{d_{0}+d / n}, \frac{\delta}{f}\right), & d>n\left(\frac{R+r+\delta}{2 r+\delta} f+d_{0}\right),\end{array}\right.$

where $r$ and $R$ are the radii of the fiber bundle and the lens, respectively, $\delta$ is the distance between the fiber core and the upper edge of the bundle, $f$ is the lens focal length, $d_{0}$ is the distance from the front of the sample to the lens center, $d$ is the depth of the scattering object, and $n$ is the sample's refractive index. Equation (3) indicates that below a threshold depth, full-angle detection can be achieved. For our probe, Fig. 4(b) shows that signals with full angular range and intensity are efficiently collected from within $350 \mu \mathrm{m}$ of mirror offset.

Again we note that, owing to the Snell's law description of the refraction at the sample surface, the sample site 1 at a physical depth of $D$ produces an angular distribution comparable to a mirror measurement at depth $D / n$ (site $1^{\prime}$ ) in air with $n$ being the sample's refractive index. Hence, the range of angles received in the mirror experiment at a depth of $350 \mu \mathrm{m}$ translates to the same range of angles that would be received at a depth of $470 \mu \mathrm{m}$ in tissue $(n=1.35)$, enough to detect the full thickness of the epithelial layer of tissue (200-300 $\mu \mathrm{m})$.

\subsection{Dispersion Compensation}

The fiber bundle has some unique optical properties that are important to its application in LCI and will be discussed in this and the following subsections. In this a/LCI system, dispersion is introduced by the variety of fibers involved, in particular, the fiber bundle. Various dispersion compensation techniques have been developed in the past, based on either hardware or numerical correction. ${ }^{34-36}$ We employ the latter approach with a second-order dispersion compensation. Let $X(k, \theta)$ denote the cross-correlation term in Eq. (1) in wavenumber domain. Dispersion compensation is performed as $\operatorname{HT}[X(k, \theta)] e^{j\left(a k^{2}+b k\right)}$, where $\mathrm{HT}(\cdot)$ is Hilbert transform and $a$ and $b$ are the secondand first-order compensation parameters.

Figure 5 shows a mirror signal with a depth resolution of $26 \mu \mathrm{m}$ in air after compensation. Compared to the theoretical value, $2 \ln 2 \lambda^{2} /(\pi \Delta \lambda) \approx 16 \mu \mathrm{m}$, the discrepancy may come primarily from the high-order ( $\geq 3$ rd) dispersion of the fiber bundle with a possible minor contribution from aberrations in the drum lens. We note that further improvements in dispersion compensation are not crutial for the current a/LCI study for the following reasons: (i) quadratic compensation is able to correct most dispersion; (ii) dispersion is not the only factor governing depth resolution for a fiber-bundle-based probe, as discussed later; and (iii) with a/LCI, we intend to measure average nucleus size for a population of cells and, therefore, an adequately large volume or depth is preferred. In this study, it is sufficient to use the same quadratic compensation for all fiber bundle pixels.

Figure 6 shows dispersion compensation results with the unprocessed depth-resolved angular scattering of a cover slip positioned in front of the probe tip [Fig. 6(a)] and the restored image after dispersion compensation where multiple surfaces can be identified [Fig. 6(b)]. The first surface seen is the sample-side surface of the lens, which strongly reflects the light to high angles due to the lack of an antireflection (AR) coating. If necessary, it could be reduced by the use of a properly AR-coated lens. The second surface is the inner surface of the optical window, which is almost invisible because the preassembling cleaning minimizes its scattering. Its signal is hence dominated by the adjacent lens surface. The other three visible surfaces-the outer surface of the optical window, the front and back surfaces of the sample coverslip-are intentionally made visible by slight finger touches to enhance scattering. The measured thicknesses of the two cover slips are consistent with the calculated value of $150 \times 1.5=225 \mu \mathrm{m}$.

The surfaces in Fig. 6(b) exhibit a curved field. The center of the fiber bundle appear $\sim 250 \mu \mathrm{m}$ (optical pathlength) deeper than the edges. A possible cause of this variation is a refractive index gradient in the bundle arising during the manufacturing process. Another possibility could be the variation of the physical length of the individual fibers.

Nonetheless, the field curvature can be flattened by numerically shifting each horizontal line. The operation is implemented together with the dispersion-compensation process by adding an extra angle-dependent phase term, $e^{-j L(\theta) k}$, with $L(\theta)$ being the relative optical depth offset at angle $\theta$. It results in $\operatorname{HT}[X(k, \theta)] e^{j\left[a k^{2}+b k-L(\theta)\right]}$, the Fourier transform of which is then shifted by $L(\theta)$ to remove the curvature. To determine $L(\theta)$, solutions of small microspheres (e.g., $260 \mathrm{~nm}$ ) are used to generate nearly uniform scattering across the entire angular range, which makes the interface of the sample clearly visible. $L(\theta)$ can then be determined by calculating the correlation between the sample fields at $\theta_{0}$ and $\theta$ and is plotted in Fig. 6(b), with the corrected field shown in Fig. 6(c).

The accuracy of the field curvature correction could be another factor affecting the depth resolution achieved with this probe if the correction is not carried out carefully. Figure 6(c) shows that, after the correction, the depth variation of each surface is insignificant compared to the peak width, indicating the correction is sufficiently accurate such as there is no influence on depth resolution. 

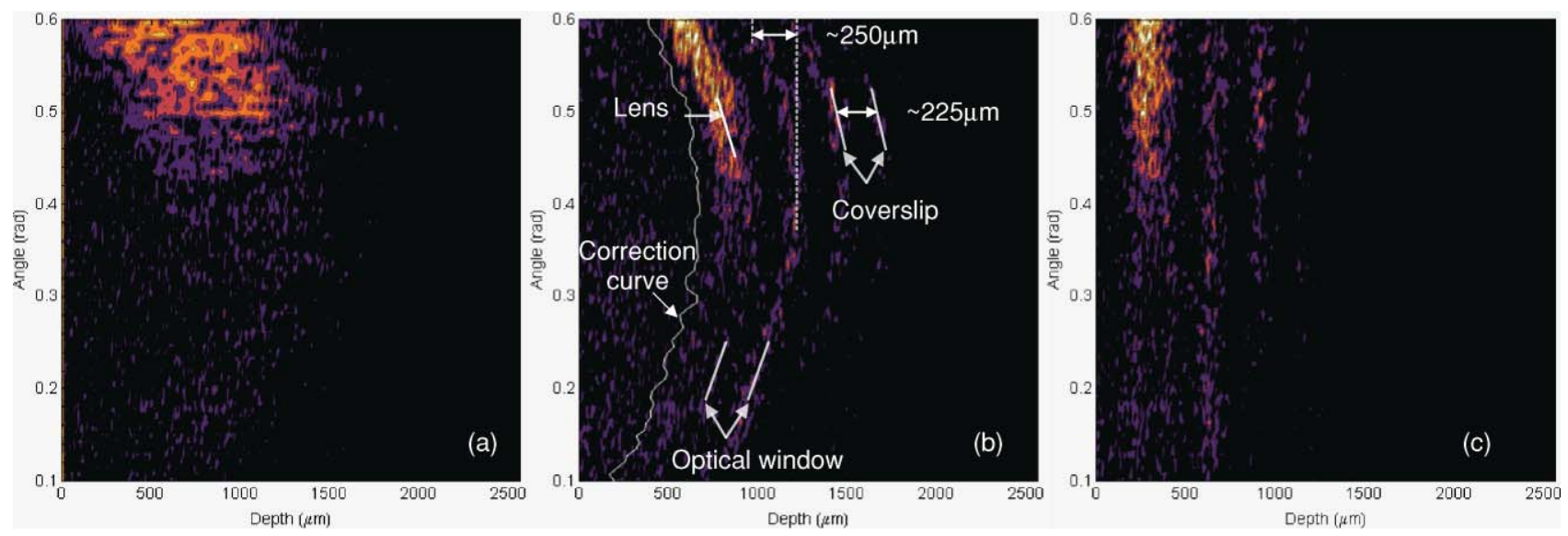

Fig. 6 Depth-resolved angular scattering from a cover slip: (a) Before and (b) after dispersion compensation, (c) field flattening by introducing extra phase shift to each angular channel.

\subsection{Interpixel Averaging}

In the spectrograph, each $\mathrm{CCD}$ pixel can receive signals from multiple fiber pixels. If the multiple fibers differ in path length, then this effect would influence the achieved depth resolution. In our system, the bundle has $\sim 160$ fiber pixels across its diameter, which are imaged onto $85 \mathrm{CCD}$ rows. Therefore, each row, on average, represents the summed interference signal from two adjacent fiber pixels. Figure 7 shows a dispersion-compensated mirror signal from a single row. Two peaks are evident with a separation of $\sim 22 \mu \mathrm{m}$, causing an effective broadening of the apparent peak width. For applications requiring higher depth resolution, this effect can be avoided by increasing the magnification of the $4 f$ imaging system to reduce the number of fiber pixels received per CCD row. The trade-off is that a higher number of CCD pixels is required to acquire scattering data from the full angular range.

\subsection{Intermodal Interference}

Another property worth discussing is the multimode operation of the fiber bundle due to its large numerical aperture. We note that higher order modes travel at different velocity through the

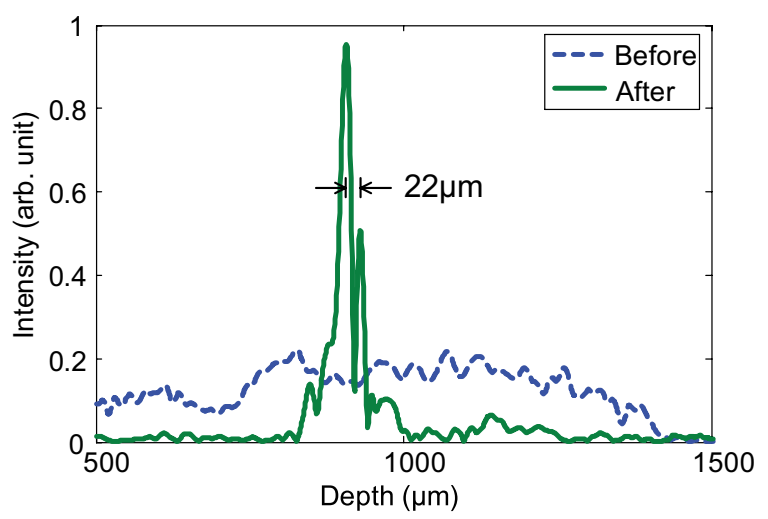

Fig. 7 Dispersion compensated mirror signal from a single CCD row showing signals from two neighboring fiber pixels. The $22 \mu \mathrm{m}$ peak-topeak separation indicates the path length difference between the two pixels. multimode fiber and hence can be path-length resolved by the LCI detection scheme. When the reference field is matched to the fundamental mode (i.e., the lowest-order mode in an optical fiber or the only mode allowed in a single-mode fiber), higherorder modes are offset by several millimeters, which is beyond the probing range of the system. ${ }^{32,37}$ In addition to path-length concerns, the energy distribution among the modes plays an important part in suppressing intermodal crosstalk. As indicated in Fig. 1(b), sample scattering is focused into the fibers with nearly normal incidence, which allows most energy to be coupled into the fundamental mode. Indeed, no high-order signal is experimentally observable within a $20-\mathrm{mm}$ range around the matching position of the reference arm. This result confirms (i) the LCI system detects the fundamental mode traveling through the fibers in the bundle, and (ii) minimal energy has been coupled into higher-order modes. Hence, for the current a/LCI system, intermodal interference is not found to be a critical issue.

\subsection{Pathlength Stability}

Another relevant property of the fiber probe is the stability of the path length due to various factors, such as bending and ambient temperature. In general, the path length of a fiber can be changed when subjected to strain or temperature variation. In laboratory experiments, we randomly place and bend the probe under normal operation conditions and record no observable change of its path length. This result can be attributed to the strain relief connector used to isolate the fiber bundle from bend-induced strain, shown in Fig. 2(b). The PEEK sheathing sustains the strain while the fiber bundle is free to move inside. The probe sheathing is also responsible for the insensitivity to room-temperature variation. Indeed, experiments that immersed the gastroscope with the probe in its accessory channel in a warm bath $\left(37^{\circ} \mathrm{C}\right)$, showed no observed changes in path length. In clinical testing, small pathlength variations of up to $100 \mu \mathrm{m}$ are observed, possibly due to mechanical and temperature influences. These variations are small and can be automatically corrected for using the lens reflection as a marker.

To summarize the probe characterization, we have discussed the optical properties of the bundle and the factors that affect the depth resolution of the system. Methods for further 

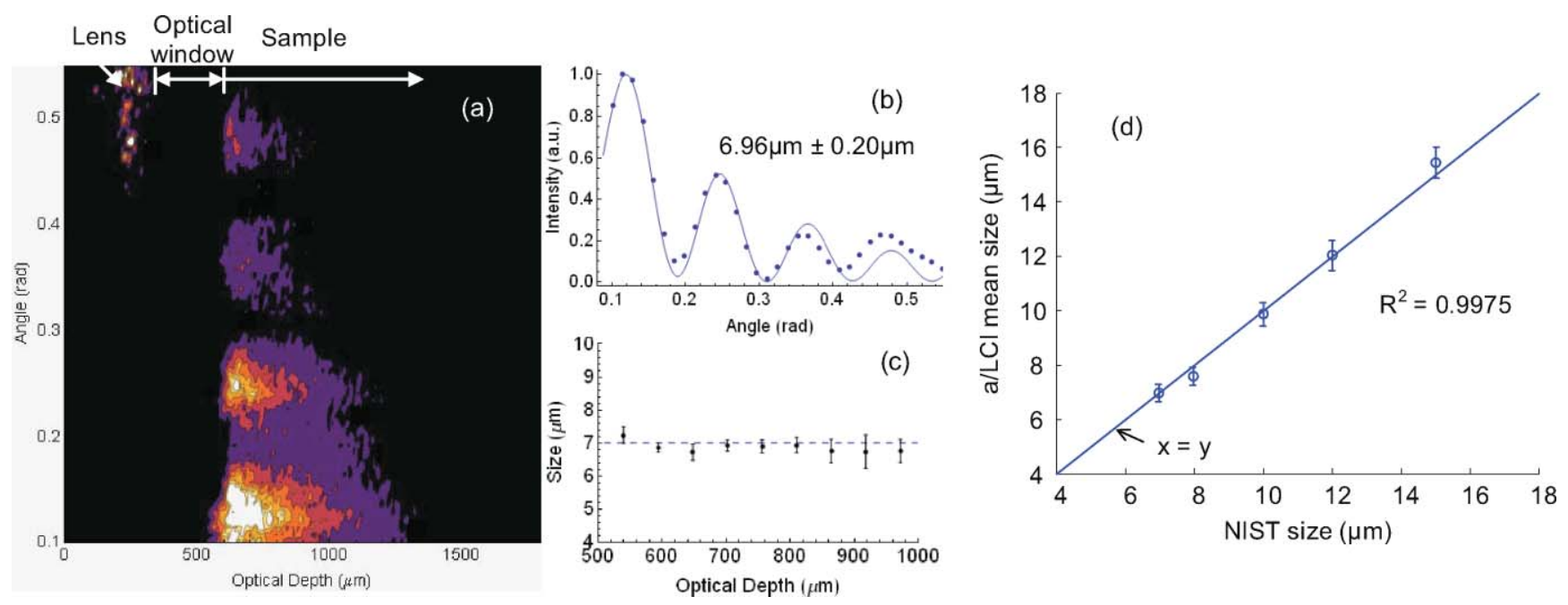

Fig. 8 Single-size microsphere phantom test: (a) Depth-resolved angular scattering distribution of $7 \mu \mathrm{m}$ microspheres, (b) Mie theory fit of angular distribution, (c) depth dependence of sizing results, and (d) a/LCl sizing results of microspheres with NIST sizes.

improvements, if necessary, were also suggested. In our current system, rather than characterizing these factors individually, we determine the effective depth resolution from the measured scattering intensity. In Section 5, we show that the effective depth resolution for this system is $26 \mu \mathrm{m}$ in tissue, which is sufficient for our purpose of measuring the average nucleus size in the epithelial layer.

\section{Phantom Study}

The performance of the a/LCI fiber probe is evaluated with measurements of both single- and double-layer polystyrene microsphere phantoms. The microspheres (Duke Scientific; Palo Alto, CA $n=1.59$ ) are suspended in a density-matching mixture of $80 \%$ water and $20 \%$ glycerol $(n=1.36)$ to produce a phantom with a mean-free-scattering path of $500 \mu \mathrm{m}$.

\subsection{Single-Size Phantom}

Phantom testing using single-sized microspheres is a standard procedure to assess the performance of a/LCI systems. ${ }^{32}$ Figure 8(a) shows a typical depth-resolved angular scattering distribution measured by immersing the probe tip into a solution of $7 \mu \mathrm{m}$ (mean diameter: $6.982 \pm 0.055 \mu \mathrm{m}$, NIST traceable) microspheres. By fitting the angular oscillations within the first $50 \mu \mathrm{m}$ of the sample to Mie theory as shown in Fig. 8(b), the scatterer size is determined to be $6.96 \pm 0.20 \mu \mathrm{m}$. The depthdependent sizing results for the first $500-\mu \mathrm{m}$ optical depth of the
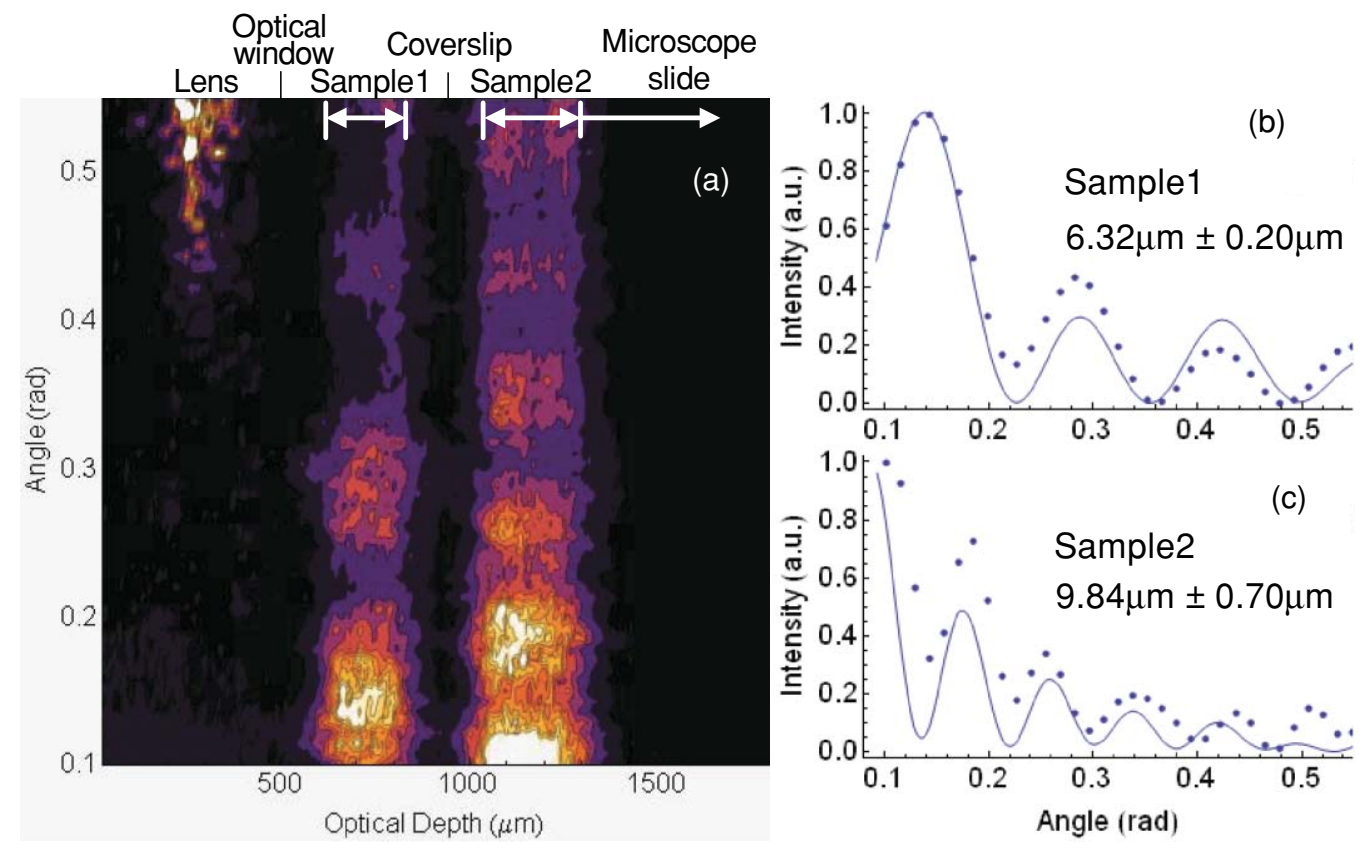

Fig. 9 Double-layer phantom testing: (a) Angular scattering distribution of 6- and 10- $\mu \mathrm{m}$ microspheres, (b) Fitting results of 6- $\mu \mathrm{m}$ microsphere using Mie theory, and (c) 10- $\mu \mathrm{m}$ microsphere results. 
sample are also shown in Fig. 8(c), demonstrating that subwavelength sizing accuracy is obtained across this range of depths. Figure $8(\mathrm{~d})$ shows that the a/LCI sizing results are in good agreement with NIST traceable sizes for 6.98-, 7.98-, 10.00-, 12.01-, and $15.02-\mu \mathrm{m}$ microspheres.

\subsection{Double-Layer Phantom}

A double-layer phantom was also prepared to demonstrate depth-resolved analysis of differently sized scatterers. The phantom consists of a chamber sandwiched by a coverslip and a microscope slide. When the microsphere-filled chamber is brought to the proximity of the probe tip, the space inbetween is filled with a second microsphere solution. Figure 9(a) shows the scattering distribution of a 100- $\mu \mathrm{m}$-thick, 6- $\mu \mathrm{m}$ microsphere layer (mean diameter: $5.990 \pm 0.045 \mu \mathrm{m}$, NIST traceable) followed by a $150 \mu \mathrm{m}$-thick $10-\mu \mathrm{m}$ microsphere layer (mean diameter: $10.00 \pm 0.05 \mu \mathrm{m}$, NIST traceable).

The average angular distribution within a $50-\mu \mathrm{m}$ layer in the middle of each sample is fit to Mie theory, and the diameters of these two samples are determined to be $6.32 \pm 0.20$ and $9.84 \pm 0.70 \mu \mathrm{m}$, respectively. These results are in excellent agreement with the sample specification and demonstrate the a/LCI's ability to achieve good fitting with multilayer samples up to $700 \mu \mathrm{m}$ of optical depth ( $\sim 500 \mu \mathrm{m}$ of physical depth).

\section{Human In Vivo Feasibility Measurement}

The length $(2.3 \mathrm{~m})$ and diameter $(2.3 \mathrm{~mm})$ of the probe allows it to be deployed through the 2.8 -mm accessory channel of a standard gastroscope for in vivo clinical evaluation of the device and the a/LCI technique. In addition, the minimized system footprint and the fast acquisition time ( $0.4 \mathrm{~s} /$ frame) have made the use of the system viable in a clinical setting. Fast acquisition reduces the system's susceptibility to motion artifacts introduced by the remote manipulation of the probe through a gastroscope as well as the inherent motion of the patient due to respiration and other involuntary processes. The ease of use allows the clinical a/LCI system to be combined with standard surveillance biopsy procedures for BE patients, without greatly increasing the time required in the procedure room. This compatibility with currently existing procedures has allowed a pilot feasibility test to be undertaken. BE patients undergoing endoscopic screening for dysplasia were recruited for this test. Informed consent was obtained from all patients. The experiment protocol was approved by the Institutional Review Board of University of North Carolina at Chapel Hill. In this section, we demonstrate the in vivo application of the a/LCI technique with examples of clinically obtained data.

\subsection{Clinical Data Acquisition}

In order to collect in vivo light-scattering information from esophageal epithelium, the a/LCI fiber probe is fed by the endoscopist through the accessory channel of the gastroscope once it has been deployed [Fig. 10(a)]. By manipulating the fiber probe and flexible tip of the gastroscope as one would do with a set of biopsy forceps, the clinician positions the probe tip in contact with the tissue of interest and applies gentle pressure in order to create a flat tissue surface from which to take measurements. The tissue becomes stretched across the probe face, resulting in consistent measurement geometry. While the clinician applies pressure, multiple acquisitions are taken of the same biopsy site by the clinical a/LCI device, after which the fiber probe is removed from the accessory channel. Following withdrawal of the a/LCI probe, a set of biopsy forceps are inserted into the accessory channel and a standard biopsy is taken at the same site of the optical biopsy, located by a transient depression in the tissue from the pressure of the probe tip. For each patient, this process is repeated at three to six clinically relevant biopsy sites selected by the clinician. Following extraction, the biopsies are fixed, stained, and analyzed by a pathologist to determine a diagnosis for the tissue in question. Figure 10(b) shows the image of one H\&E stained biopsy from the squamocolumnar junction.

\subsection{Clinical Data Processing}

Once acquired, the data are processed with an automatic a/LCI processing script to determine the depth-resolved nuclear sizes and densities for each tissue layer, ${ }^{29}$ corresponding to $50 \mu \mathrm{m}$ in physical depth. The starting depth is determined by analyzing the depth profile, obtained by summing the data across all the angular channels of the a/LCI scan, as shown in Fig. 10(c).
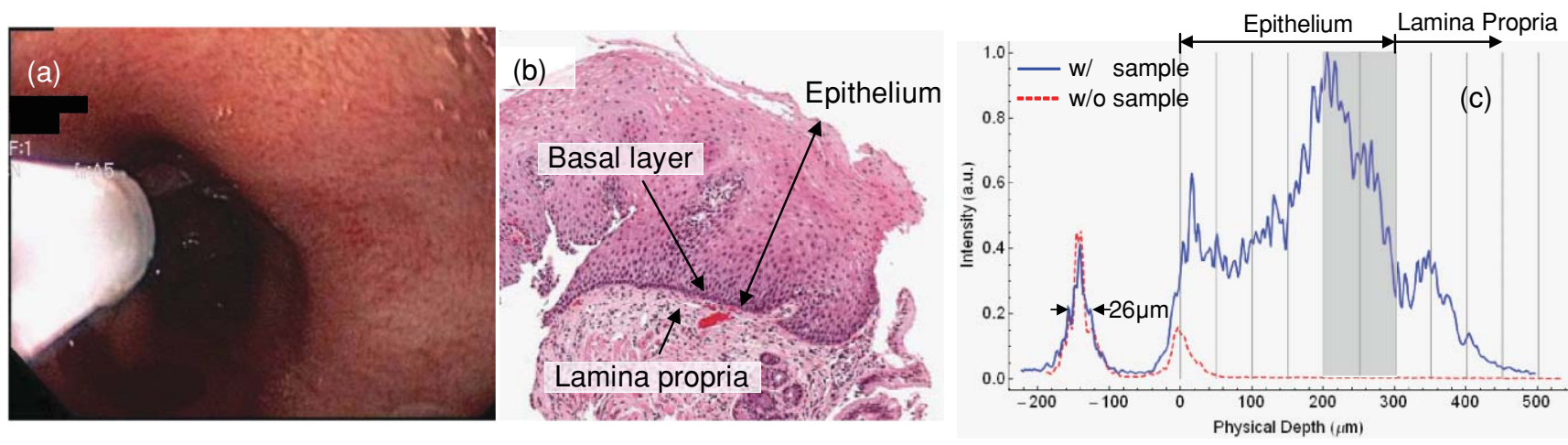

Fig. 10 In vivo data collection using the a/LCl fiber probe: (a) Endoscopic photograph of the a/LCl probe deployed in human esophagus, (b) light microscopy image of $\mathrm{H} \& \mathrm{E}$ stained biopsy from the squamocolumnar junction, and (c) depth profiles obtained by summing across all angular channels without (dashed line) and with (solid line) the presence of tissue. The gray lines indicate the depth bins for processing, and the gray bar estimates the basal layer. 


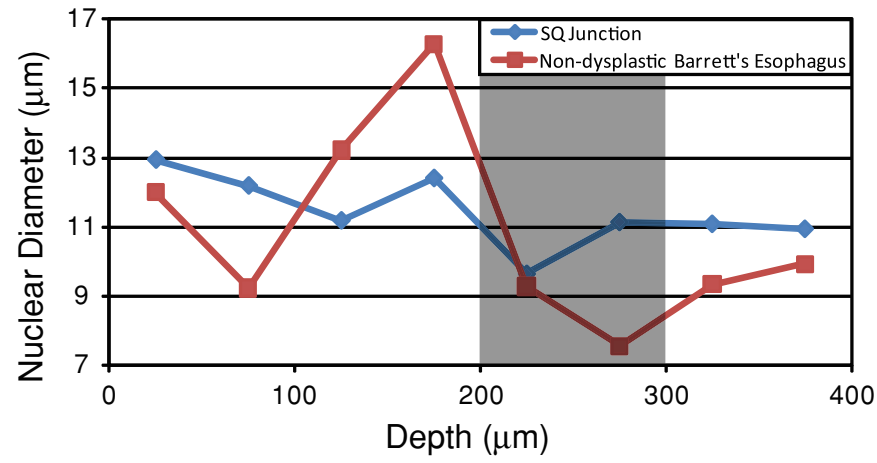

(a)

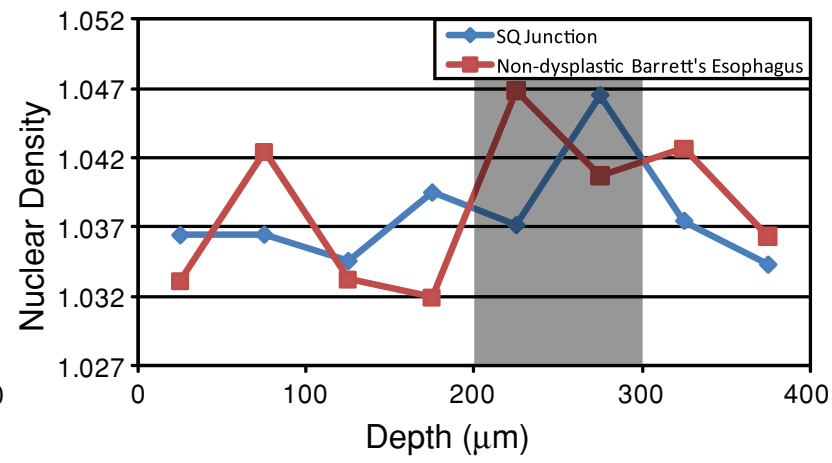

(b)

Fig. 11 (a) Nuclear sizing and (b) density information for the clinical depth scan of normal squamocolumnar junction tissue (diamond) and BE tissue (square). Gray area estimates the basal layer.

With no sample present (dashed line), the depth profile reveals two peaks, one from the lens surface and the other from the outer surface of the optical window. Both have a FWHM of $26 \mu \mathrm{m}$, which is the actual physical depth resolution achieved with the probe after taking into account the effects of high-order dispersion, field-flattening error, and interpixel averaging. The $50-\mu \mathrm{m}$ bin size has been chosen to average over a larger tissue volume.

The second surface of the optical window is used to determine the topmost surface of the sample and remains constant throughout the remaining scans. Analysis is executed for the first $500 \mu \mathrm{m}$ of the tissue, which is consistent with the effective depth range of the probe. This range is sufficient to capture the entire epithelial layer of the tissue, including the diagnostically relevant basal layer, which appears $200-300 \mu \mathrm{m}$ into the epithelium. Figure 10(c) also shows the depth profile obtained when probing a tissue in vivo (solid line). The signal from the outer surface of the optical window is not individually indentified due to the contact with the tissue but its position can be determined relative to the first surface.

\subsection{Clinical Sizing Results}

Figure 11(a) shows the nuclear sizing information obtained from the clinical scan for the first $400 \mu \mathrm{m}$ of tissue taken from the same site in Fig. 10(b). The histopathological diagnosis for this particular tissue biopsy indicates that the tissue biopsy is from the squamocolumnar junction with no evidence of intestinal metaplasia (Barrett's esophagus) or dysplasia. Unique sizing information is obtained from each depth layer of the tissue, indicating the ability of the clinical a/LCI system to retrieve depth-resolved in vivo morphological and optical information from the tissue in question. We note that the pattern observed of a decreasing nuclear size across the epithelial layer is similar to that observed for squamous tissue in Ref. 29, with the reading at the basal layer (gray box), indicating a nuclear size and density measurement consistent with the histopathological diagnosis of a normal tissue type. For comparison, Fig. 11(a) also shows the results for a biopsy diagnosed with nondysplastic BE from the same patient, where the pattern of nuclear sizes reaches a maximum between the surface and basal layers of the epithelium, similar to that observed for gastric tissue in Ref. 29. These depth-resolved sizing patterns are closely associated with the pathological tissue conditions and can serve as a basis for using the a/LCI probe for potential assessment of tissue health. Further clinical studies will seek to better define the ability of a/LCI to discriminate between tissue types. Figure 11(b) shows the nuclear density information for these two biopsies.

\section{Summary}

In this paper, we demonstrate a Fourier-domain a/LCI system suitable for in vivo clinical studies. The key technical advance of this system is an endoscopic fiber probe, which is compatible with the standard accessory channel of a gastroscope. We demonstrate that this probe can be used to collect scattered light from the in vivo human esophageal epithelium, which is analyzed to obtain depth-resolved structural information. Validation experiments using tissue phantoms demonstrate accurate analysis of scattering objects with subwavelength accuracy in multiple depth-resolved layers. To enable clinical application, the fiber probe features a long probe length and small probe diameter, while the system includes a compact footprint and subsecond data acquisition. The optical characteristics of the fiber probe have been analyzed in detail and the results may provide useful information for other LCI techniques that would incorporate coherent fiber bundles. In vivo measurements from a clinical testing in BE patients have been presented to demonstrate the ability of a /LCI to obtain depth-resolved nuclear morphological information and point the way to future clinical studies.

\section{Acknowledgments}

Adam Wax is a founder of Oncoscope, Inc. This work was supported in part by the National Institutes of Health (National Cancer Institute) under Grant No. R33-CA 109907 and a grant from the Coulter Translational Partnership.

\section{References}

1. N. J. Shaheen and J. E. Richter, "Barrett's oesophagus," Lancet 373(9666), 850-861 (2009).

2. R. E. Sampliner, "Updated guidelines for the diagnosis, surveillance, and therapy of Barrett's esophagus," Am. J. Gastroenterol. 97(8), 1888-1895 (2002).

3. H. Stepp, R. Sroka, and R. Baumgartner, "Fluorescence endoscopy of gastrointestinal diseases: Basic principles, techniques, and clinical experience," Endoscopy 30(4), 379-386 (1998). 
4. P. Sharma, A. Bansal, S. Mathur, S. Wani, R. Cherian, D. McGregor, A. Higbee, S. Hall, and A. Weston, "The utility of a novel narrow band imaging endoscopy system in patients with Barrett's esophagus,' Gastrointest. Endosc. 64(2), 167-175 (2006).

5. K. Gono, T. Obi, M. Yamaguchi, N. Ohyama, H. Machida, Y. Sano, S. Yoshida, Y. Hamamoto, and T. Endo, "Appearance of enhanced tissue features in narrow-band endoscopic imaging," J. Biomed. Opt. 9(3), 568-577 (2004).

6. A. M. Buchner and M. B. Wallace, "Future expectations in digestive endoscopy: competition with other novel imaging techniques," Best Pract. Res. Clin. Gastroenterol. 22(5), 971-987 (2008).

7. B. C. Wilson, "Detection and treatment of dysplasia in Barrett's esophagus: a pivotal challenge in translating biophotonics from bench to bedside," J. Biomed. Opt. 12(5), 051401 (2007).

8. J. Haringsma, "Barrett's oesophagus: New diagnostic and therapeutic techniques," Scand. J. Gastroenterol. 37, 9-14 (2002).

9. L. T. Perelman, V. Backman, M. Wallace, G. Zonios, R. Manoharan, A. Nusrat, S. Shields, M. Seiler, C. Lima, T. Hamano, I. Itzkan, J. Van Dam, J. M. Crawford, and M. S. Feld, "Observation of periodic fine structure in reflectance from biological tissue: A new technique for measuring nuclear size distribution," Phys. Rev. Lett. 80(3), 627-630 (1998).

10. V. Backman, M. B. Wallace, L. T. Perelman, J. T. Arendt, R. Gurjar, M. G. Muller, Q. Zhang, G. Zonios, E. Kline, T. McGillican, S. Shapshay, T. Valdez, K. Badizadegan, J. M. Crawford, M. Fitzmaurice, S. Kabani, H. S. Levin, M. Seiler, R. R. Dasari, I. Itzkan, J. Van Dam, and M. S. Feld, "Detection of preinvasive cancer cells," Nature 406(6791), 35-36 (2000).

11. M. B. Wallace, L. T. Perelman, V. Backman, J. M. Crawford, M. Fitzmaurice, M. Seiler, K. Badizadegan, S. J. Shields, I. Itzkan, R. R. Dasari, J. Van Dam, and M. S. Feld, "Endoscopic detection of dysplasia in patients with Barrett's esophagus using light-scattering spectroscopy," Gastroenterology 119(3), 677-682 (2000).

12. I. Georgakoudi, B. C. Jacobson, J. Van Dam, V. Backman, M. B. Wallace, M. G. Muller, Q. Zhang, K. Badizadegan, D. Sun, G. A. Thomas, L. T. Perelman, and M. S. Feld, "Fluorescence, reflectance, and lightscattering spectroscopy for evaluating dysplasia in patients with Barrett's esophagus," Gastroenterology 120(7), 1620-1629 (2001).

13. C. Lau, O. Scepanovic, J. Mirkovic, S. McGee, C.-C. Yu, S. Fulghum, M. Wallace, J. Tunnell, K. Bechtel, and M. Feld, "Re-evaluation of model-based light-scattering spectroscopy for tissue spectroscopy," $J$. Biomed. Opt. 14(2), 024031 (2009).

14. L. B. Lovat, K. Johnson, G. D. Mackenzie, B. R. Clark, M. R. Novelli, S. Davies, M. O'Donovan, C. Selvasekar, S. M. Thorpe, D. Pickard, R. Fitzgerald, T. Fearn, I. Bigio, and S. G. Bown, "Elastic scattering spectroscopy accurately detects high grade dysplasia and cancer in Barrett's oesophagus," Gut 55(8), 1078-1083 (2006).

15. A. Dhar, K. S. Johnson, M. R. Novelli, S. G. Bown, I. J. Bigio, L. B. Lovat, and S. L. Bloom, "Elastic scattering spectroscopy for the diagnosis of colonic lesions: initial results of a novel optical biopsy technique," Gastrointest. Endosc. 63(2), 257-261 (2006).

16. L.-M. Wong Kee Song, A. Molckovsky, K. K. Wang, L. J. Burgart, B. Dolenko, R. L. Somorjai, and B. C. Wilson, "Diagnostic potential of Raman spectroscopy in Barrett's esophagus," Proc. SPIE 5692, 140-146 (2005).

17. M. G. Shim, L. Song, N. E. Marcon, and B. C. Wilson, "In vivo nearinfrared Raman spectroscopy: Demonstration of feasibility during clinical gastrointestinal endoscopy," Photochem. Photobiol. 72(1), 146-150 (2000).

18. U. Utzinger and R. R. Richards-Kortum, "Fiber optic probes for biomedical optical spectroscopy," J. Biomed. Opt. 8(1), 121-147 (2003).

19. A. L. Polglase, W. J. McLaren, S. A. Skinner, R. Kiesslich, M. F. Neurath, and P. M. Delaney, "A fluorescence confocal endomicroscope for in vivo microscopy of the upper- and the lower-GI tract," Gastrointest. Endosc. 62(5), 686-695 (2005).

20. E. S. Barhoum, R. S. Johnston, and E. J. Seibel, "Optical modeling of an ultrathin scanning fiber endoscope, a preliminary study of confocal versus non-confocal detection," Opt. Express 13(19), 7548-7562 (2005).
21. K. Murakami, "A miniature confocal optical scanning microscope for endoscope," Proc. SPIE, 5721, 119-131 (2005).

22. V. Becker, T. Vercauteren, C. H. von Weyhern, C. Prinz, R. M. Schmid, and A. Meining, "High-resolution miniprobe-based confocal microscopy in combination with video mosaicing (with video)," Gastrointest. Endosc. 66(5), 1001-1007 (2007).

23. A. Meining, D. Saur, M. Bajbouj, V. Becker, E. Peltier, H. Hoefler, C. H. Von Weyhern, R. M. Schmid, and C. Prinz, "In vivo histopathology for detection of gastrointestinal neoplasia with a portable, Confocal miniprobe: an examiner blinded analysis," Clin. Gastroenterol. Hepatol. 5(11), 1261-1267 (2007).

24. H. Pohl, T. Rosch, M. Vieth, M. Koch, V. Becker, M. Anders, A. C. Khalifa, and A. Meining, "Miniprobe confocal laser microscopy for the detection of invisible neoplasia in patients with Barrett's oesophagus," Gut 57(12), 1648-1653 (2008).

25. B. J. Vakoc, M. Shishko, S. H. Yun, W. Y. Oh, M. J. Suter, A. E. Desjardins, J. A. Evans, N. S. Nishioka, G. J. Tearney, and B. E. Bouma, "Comprehensive esophageal microscopy by using optical frequencydomain imaging (with video)," Gastrointest. Endosc. 65(6), 898-905 (2007).

26. Y. Chen, A. D. Aguirre, P. L. Hsiung, S. Desai, P. R. Herz, M. Pedrosa, Q. Huang, M. Figueiredo, S. W. Huang, A. Koski, J. M. Schmitt, J. G. Fujimoto, and H. Mashimo, "Ultrahigh resolution optical coherence tomography of Barrett's esophagus: preliminary descriptive clinical study correlating images with histology," Endoscopy 39(7), 599-605 (2007).

27. W. Jung, D. T. McCormick, Y.-C. Ahn, A. Sepehr, M. Brenner, B. Wong, N. C. Tien, and Z. Chen, "In vivo three-dimensional spectral domain endoscopic optical coherence tomography using a microelectromechanical system mirror," Opt. Lett. 32(22), 3239-3241 (2007).

28. P. R. Herz, Y. Chen, A. D. Aguirre, J. G. Fujimoto, H. Mashimo, J. Schmitt, A. Koski, J. Goodnow, and C. Petersen, "Ultrahigh resolution optical biopsy with endoscopic optical coherence tomography," Opt. Express 12(15), 3532-3542 (2004).

29. W. J. Brown, J. W. Pyhtila, N. G. Terry, K. J. Chalut, T. A. D'Amico, T. A. Sporn, J. V. Obando, and A. Wax, "Review and recent development of angle-resolved low-coherence interferometry for detection of precancerous cells in human esophageal epithelium," IEEE J. Sel. Top. Quantum Electron. 14(1), 88-97 (2008).

30. A. Wax, C. H. Yang, V. Backman, K. Badizadegan, C. W. Boone, R. R. Dasari, and M. S. Feld, "Cellular organization and substructure measured using angle-resolved low-coherence interferometry," Biophys. J. 82(4), 2256-2264 (2002).

31. K. J. Chalut, J. H. Ostrander, M. G. Giacomelli, and A. Wax, "Light scattering measurements of subcellular structure provide noninvasive early detection of chemotherapy-induced apoptosis," Cancer Res. 69(3), 1199-1204 (2009).

32. J. W. Pyhtila, J. D. Boyer, K. J. Chalut, and A. Wax, "Fourierdomain angle-resolved low coherence interferometry through an endoscopic fiber bundle for light-scattering spectroscopy," Opt. Lett. 31(6), 772-774 (2006)

33. J. W. Pyhtila, K. J. Chalut, J. D. Boyer, J. Keener, T. D’Amico, M. Gottfried, F. Gress, and A. Wax, "In situ detection of nuclear atypia in Barrett's esophagus by using angle-resolved low-coherence interferometry," Gastrointest. Endosc. 65(3), 487-491 (2007).

34. A. F. Fercher, C. K. Hitzenberger, M. Sticker, and R. Zawadzki, "Numerical dispersion compensation for partial coherence interferometry and optical coherence tomography," Opt. Express 9(12), 610-615 (2001).

35. J. Wojtkowski, V. J. Srinivasan, T. H. Ko, J. G. Fujimoto, A. Kowalczyk, and J. S. Duker, "Ultrahigh-resolution, high-speed, Fourier domain optical coherence tomography and methods for dispersion compensation," Opt. Express 12(11), 2404-2422 (2004).

36. D. Choi, H. Hiro-Oka, T. Amano, H. Furukawa, F. Kano, M. Nakanishi, K. Shimizu, and K. Ohbayashi, "Numerical compensation of dispersion mismatch in discretely swept optical-frequency-domain-reflectometry optical coherence tomography," Jpn. J. Appl. Phys. 45(7), 6022-6027 (2006).

37. T. Xie, D. Mukai, S. Guo, M. Brenner, and Z. Chen, "Fiber-opticbundle-based optical coherence tomography," Opt. Lett. 30(14), 1083-1085 (2005). 